\title{
ANALYSIS OF POWER TRANSFORMATOR CONDITIONS USING DGA METHOD USING ARTIFICIAL NEURAL NETWORK IN KRAKATAU ELECTRICAL POWER COMPANY
}

\author{
Hartono $^{* 1}$, Y. Muharni ${ }^{2}$, C. Adipura ${ }^{3}$, W. Martiningsih ${ }^{4}$, M. Otong ${ }^{5}$, M. Irvan ${ }^{6}$ \\ ${ }^{* 1}, 2,3,4,5,6$ Department of Electrical Engineering, Universitas Sultan Ageng Tirtayasa, Indonesia
}

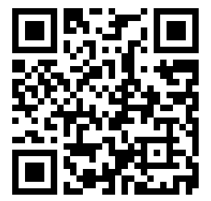

DOI: https://doi.org/10.29121/ijetmr.v7.i6.2020.572

Article Citation: Hartono, Y.

Muharni, C. Adipura, W.

Martiningsih, M. Otong, and M. Irvan. (2020). ANALYSIS OF POWER

TRANSFORMATOR CONDITIONS

USING DGA METHOD USING

ARTIFICIAL NEURAL NETWORK IN

KRAKATAU ELECTRICAL POWER

COMPANY. International Journal of

Engineering Technologies and

Management Research, 7(6), 77-88.

https://doi.org/10.29121/ijetmr.v7

i6.2020.572

Published Date: 16 June 2020

Keywords:

Dissolved Gas Analysis (DGA)

IEC Ratio Method

Artificial Neural Network (ANN)

\section{ABSTRACT}

Test method that can be done for transformer oil with DGA method. In identifying early transformer conditions, one of them is using IEC 60599 Standards. The artificial neural network training process used 341 data in the presence of nine conditions based on the IEC standard. The best network architecture configuration is a configuration with 3 neurons in the input layer, 10 neurons in the first hidden layer, 20 neurons in the second hidden layer, 20 neurons in the third hidden layer and 4 neurons in the output layer with the transfer logic. The results of the training give a regression value of 0.95216 and MSE (Mean Square Error) is worth 0.000216. Testing of artificial neural networks is done 19 first test data is performed to determine the number of transformer conditions that can be diagnosed by each method. From the test data obtained the accuracy value for artificial neural network models is $94.7 \%$.

The following will guide the structure of your abstract:

Motivation/Background: Using the neural network method in this study is expected to improve accuracy and improve the transformer analysis process. Transformer to make one effective and fast way for transformers.

Method: The IEC method is an effective method for implementing transformers. The way this method works is by comparing the concentration of solute, then the results are represented into nine kinds of conditions. However, this method has a weakness that is the length of time in the analysis process. Therefore, to overcome these deficiencies, this study uses the Artificial Neural Network (ANN) method with a comparison of the use of gas as its input and the condition transformer as its target.

Results: The results of the training give a regression value of 0.95216 and MSE (Mean Square Error) is worth 0.000216.

Conclusions: This study uses 460 data from existing data into 2 namely data for training that brings 341 data and data for testing to get 19 data. In this study using a neural network resolves the problem in this study. in this study obtained an accuracy of $94.4 \%$, so this artificial neural network method has good potential to assist in this study. 
Analysis of Power Transformator Conditions Using DGA Method Using Artificial Neural Network in Krakatau Electrical Power Company

\section{INTRODUCTION}

One form of transformer maintenance is by conducting tests to determine the state of the transformer. Tests carried out to test insulating oil in addition to translucency testing and dielectric gain-loss testing, PLN also applies the DGA (Dissolve Gas Analysis) test method. This test method is carried out to test the condition of the insulating oil by taking insulating oil samples from the transformer unit to determine the types of gas dissolved in the transformer oil. The purpose of DGA testing is the transformer to be known, Therefore it is necessary to do an analysis for abnormalities in the transformer by testing DGA (Dissolved Gas Analysis) so that it can be known in advance about the likelihood of the transformer [15].

When the transformer works in normal conditions, there are various kinds of gas produced in small amounts, called $\mathrm{C} 2 \mathrm{H} 4, \mathrm{C} 2 \mathrm{H} 2, \mathrm{CH} 4, \mathrm{~N} 2$ and 02 . When a failure occurs in the transformer, the concentration of gas produced will vary depending on the type of failure in the transformer. The level of gas produced by the oil transformer is used as an indication of the condition of the transformer. The gases used in DGA analysis are H2 (Hydrogen), CH4 (Methane), C2H4 (Ethylene), C2H6 (Ethane), C2H2 (Acetylene), CO (Carbon monoxide), and CO2 (Carbon dioxide [12].

The dissolved gas (DGA) analysis method is an analysis of the condition of the transformer based on the amount of dissolved gas in transformer oil, by extracting the gases from oil samples taken from the transformer. The extracted gas is then added according to each gas and is calculated in ppm units (parts per million). From the results of this DGA test it can be known in advance about the failure of the transformer that may arise. There are several DGA test standards that have been determined by IEEE, including the Duval Triangle, Total Combustible Gas (TDCG), Key Gas, Roger Ratio, Doernenburg Ratio and IEC Ratio.

From several methods of data interpretation, DGA and test standards established by the IEEE, then made here using one of the test standards namely IEC Ratio. The main reason for using the IEC Ratio method is because this method is still rarely used to do DGA analysis especially in Indonesia. However, the test standard for DGA analysis also has drawbacks, the main drawback of the Ratio method is the failure method for all data.

To overcome this problem, we need a solution from the AI (Artificial Intelligence) method, one of which is ANN (Artificial Neural Network). ANN. Knowing the funds needed from the pattern and being able to acquire knowledge to buy nonlinear objects, requires quite a lot of data in the training process. But the expected method ANN is able to provide accurate and fast analysis results for reading transformers.

\section{MATERIALS AND METHODS}

Dissolved gas analysis (DGA) is an analysis of the condition of the transformer which is based on the amount of dissolved gas in transformer oil [2]. For several years the method of analyzing dissolved gases in oil has been used as a transformer diagnosis tool. Analyzing dissolved gas content requires several steps, namely taking oil samples, extracting gas, interpreting data and drawing conclusions. Dissolved gas analysis is done by measuring the total flammable gas content which is interpreted by various methods. Commonly used methods are the key gas, the roger ratio method, and the Duvall triangle method.

Roger ratio method is to compare the amount of different gases by dividing one gas with another, this forms a ratio ratio between one gas with another gas. This method uses a ratio of three gases, namely C2H2 / $\mathrm{C} 2 \mathrm{H} 4, \mathrm{CH} 4$ / $\mathrm{H} 2$ and C2H4 / C2H6. Roger ratio actually consists of 4 ratios namely C2H2 / C2H4, CH4 / H2, C2H4 / C2H6 and $\mathrm{C} 2 \mathrm{H} 6$ / CH4. However, the $\mathrm{C} 2 \mathrm{H} 6$ / $\mathrm{CH} 4$ ratio only indicates a limited temperature range from decomposition but does not help in identifying further faults. It should be noted that the roger ratio method is used for disturbance analysis rather than for detecting interference and therefore interference must be detected using the Institute of Electrical and Electronics Engineers (IEEE) limits.

Table 1: Roger Ratio

\begin{tabular}{|c|c|c|}
\hline Ratio Code & Range & Code \\
\hline $\mathrm{CH}_{4} / \mathrm{H}_{2}(\mathrm{i})$ & $<=0.1$ & 5 \\
& $>0.1<1.0$ & 0 \\
& $>=1.0<3.0$ & 1 \\
& $>=3.0$ & 2 \\
\hline $\mathrm{C}_{2} \mathrm{H}_{6} / \mathrm{CH}_{4}(\mathrm{j})$ & $<1.0$ & 0 \\
\hline
\end{tabular}


Hartono, Y. Muharni, C. Adipura, W. Martiningsih, M. Otong, and M. Irvan

\begin{tabular}{|l|c|c|}
\hline & $>=1.0$ & 1 \\
\hline $\mathrm{C}_{2} \mathrm{H}_{4} / \mathrm{C}_{2} \mathrm{H}_{6}(\mathrm{k})$ & $<1.0$ & 0 \\
& $>=1.0<3.0$ & 1 \\
& $>=3.0$ & 2 \\
\hline $\mathrm{C}_{2} \mathrm{H}_{2} / \mathrm{C}_{2} \mathrm{H}_{4}(\mathrm{l})$ & $>0.5$ & 0 \\
& $>=0.5<3.0$ & 1 \\
& $>=3.0$ & 2 \\
\hline
\end{tabular}

Table 2: Roger's Failure Diagnosis Ratio

\begin{tabular}{|c|c|c|c|c|}
\hline I & $\mathrm{J}$ & $\mathrm{K}$ & $\mathrm{L}$ & Diagnosis \\
\hline 0 & 0 & 0 & 0 & Normal \\
\hline 5 & 0 & 0 & 0 & Partial Discharge \\
\hline $1-2$ & 0 & 0 & 0 & Slight Overheating $<150^{\circ} \mathrm{C}$ \\
\hline $1-2$ & 1 & 0 & 0 & Overheating $150^{\circ} \mathrm{C}-200^{\circ} \mathrm{C}$ \\
\hline 0 & 1 & 0 & 0 & Overheating $200^{\circ} \mathrm{C}-300^{\circ} \mathrm{C}$ \\
\hline 0 & 0 & 1 & 0 & General conductor overheating \\
\hline 1 & 0 & 1 & 0 & Winding circulating currents \\
\hline 1 & 0 & 2 & 0 & Core and tank circulating currents, overheated joints \\
\hline 0 & 0 & 0 & 1 & Flashover without power follow through \\
\hline 0 & 0 & $1-2$ & $1-2$ & Arc with power follow through \\
\hline 0 & 0 & 2 & 2 & Continuous sparking to floating potential \\
\hline 5 & 0 & 0 & $1-2$ & Partial discharge with tracking (note CO) \\
\hline
\end{tabular}

IEC is one of the popular standards for determining transformer conditions based on the ratio of five key gases $\mathrm{H} 2, \mathrm{CH} 4, \mathrm{C} 2 \mathrm{H} 4, \mathrm{C} 2 \mathrm{H} 6$, and $\mathrm{C} 2 \mathrm{H} 2$ in this method of gas constellation $(\mathrm{R} 1=\mathrm{C} 2 \mathrm{H} 2 / \mathrm{C} 2 \mathrm{H} 4, \mathrm{R} 2=\mathrm{CH} 4 / \mathrm{H} 2$, and $\mathrm{R} 3=$ $\mathrm{C} 2 \mathrm{H} 4$ / $\mathrm{C} 2 \mathrm{H} 6$ ) the code of the ratio is used to determine a condition in the tansformator. The combination of each gas ratio code is used to determine the condition of the transformer after the gas with the code given in each condition. The combination of individual code X1, X2 and X3 is an indicator of the possibility of failure. Table 2 below shows the transformer failure codes based on the IEC 599 standard of the individual codes X1, X2, and X3 shown in table 3 AND 4. These gas key ratio coders can help facilitate the development of computational programming that is easier to identify transformer failures. However, this IEC ratio method in some cases, fails to identify the type of failure accurately (Shakeb A. Khan, 2014).

Table 3: Code Rules for the IEC Method [14]

\begin{tabular}{|c|c|c|c|}
\hline \multicolumn{3}{|c|}{ Gas Ratio Range } & Code \\
\cline { 1 - 3 } $\mathrm{C}_{2} \mathrm{H}_{2} / \mathrm{C}_{2} \mathrm{H}_{4}$ & $\mathrm{CH}_{4} / \mathrm{H}_{2}$ & $\mathrm{C}_{2} \mathrm{H}_{4} / \mathrm{C}_{2} \mathrm{H}_{6}$ & \\
\hline$<0.1$ & $0.1-1$ & $<1$ & 0 \\
\hline $0.1-3$ & $>0.1$ & $1-3$ & 1 \\
\hline$>3$ & $>1$ & $>3$ & 2 \\
\hline
\end{tabular}

Table 4: Failure Classification by IEC 60599 Method [14]

\begin{tabular}{|l|l|c|c|c|}
\hline No & Condition Characteristics & R1 & R2 & R3 \\
\hline 1 & Normal (N) & 0 & 0 & 0 \\
\hline 2 & PD due to low energy density & 0 & 1 & 0 \\
\hline 3 & PD due to High energy density & 1 & 1 & 0 \\
\hline 4 & D1 & $1-2$ & 0 & $1-2$ \\
\hline 5 & D2 & 1 & 0 & 2 \\
\hline 6 & TR $<150^{\circ} \mathrm{C}$ & 0 & 0 & 1 \\
\hline 7 & TR Between $150^{\circ} \mathrm{C}-300^{\circ} \mathrm{C}$ & 0 & 2 & 0 \\
\hline 8 & TR Between $300^{\circ} \mathrm{C}-700^{\circ} \mathrm{C}$ & 0 & 2 & 1 \\
\hline 9 & TT $>700^{\circ} \mathrm{C}$ & 0 & 2 & 2 \\
\hline
\end{tabular}


Analysis of Power Transformator Conditions Using DGA Method Using Artificial Neural Network in Krakatau Electrical Power Company

Neural Network (NN) is a network of a collection of small processing units that are modeled based on human neural networks. This NN is an adaptive system that can change its structure to solve the problem of external or internal information flowing through the network. The structure is very parallel, resulting in the ability to selfregulate to represent information and solve problems quickly.

In this paper a new method for Artificial Neural Networks is applied to DGA for the interpretation of initial errors in power transformers. Error interpretation can be found as a multi-class classification problem. ANN automatically adjusts network parameters, connection weights, and bias requirements of neural networks, to achieve the best model based on the proposed evolution algorithm, which provides solutions to complex classification problems, because the hidden relationship between the type of error and dissolved gas can be recognized by ANN through training process.

\section{RESULTS AND DISCUSSIONS}

To overcome these deficiencies, this study uses an artificial neural network (ANN) method with a ratio of gas as the input and condition of the transformer as the target. the gas comparison ratio is R1, R2, R3 and has nine outputs which each detect the state of the transformer. To simplify the ANN training process, the output of each condition is changed to certain numbers so that it can be understood by the ANN algorithm.

Table 5: Input and Output

\begin{tabular}{|l|c|c|c|}
\hline \multicolumn{3}{|c|}{ Input value } & Output value \\
\cline { 1 - 2 } $\begin{array}{l}\mathrm{C}_{2} \mathrm{H}_{2} / \mathrm{C}_{2} \mathrm{H}_{4} \\
(\mathrm{R} 1)\end{array}$ & $\begin{array}{c}\mathrm{CH}_{4} / \mathrm{H}_{2} \\
(\mathrm{R} 2)\end{array}$ & $\begin{array}{c}\mathrm{C}_{2} \mathrm{H}_{4} / \mathrm{C}_{2} \mathrm{H}_{6} \\
(\mathrm{R} 3)\end{array}$ & \\
\hline$<0.1$ & $0.1-1$ & $<1$ & 0001 \\
\hline$<0.1$ & $<0.1$ & $<1$ & 0010 \\
\hline $0.1-3$ & $<0.1$ & $<1$ & 0011 \\
\hline$\geq 0.1$ & $0.1-1$ & $\geq 1$ & 0100 \\
\hline $0.1-3$ & $0.1-1$ & $>3$ & 0101 \\
\hline$<0.1$ & $0.1-1$ & $1-3$ & 0110 \\
\hline$<0.1$ & $>1$ & $<1$ & 0111 \\
\hline$<0.1$ & $>1$ & $1-3$ & 1000 \\
\hline$<0.1$ & $>1$ & $>3$ & 1001 \\
\hline
\end{tabular}

In this paper the MATLAB software is used to build the ANN model. MLP neural networks are made separately for the Rogers ratio method and the IEC ratio method. Logic, and logic functions are used as transfer functions. Figure 2 shows an Artificial Neural Network with five hidden layers. For the development of neural networks, 360 sample datasets are used. 341 datasets were used for training purposes and 19 datasets were used for testing purposes. To interact with MLP networks, a GUI is created using MATLAB. It provides a user interface with the network. The value of the gas produced due to error is given as network input using the GUI as shown in figure 3. By using this panel, the method applied by ANN is selected. The error type window displays the type of error.

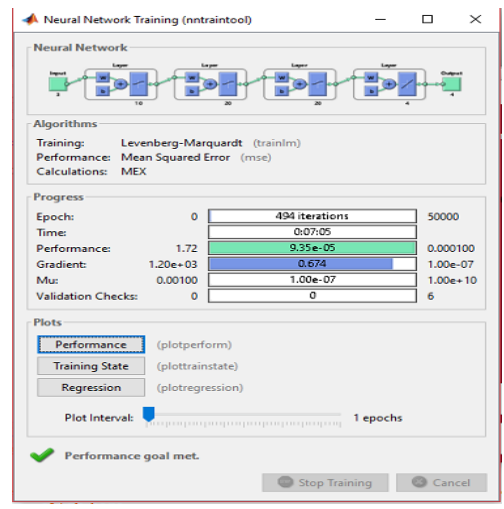

Figure 1: Artificial Neural Network 
Hartono, Y. Muharni, C. Adipura, W. Martiningsih, M. Otong, and M. Irvan

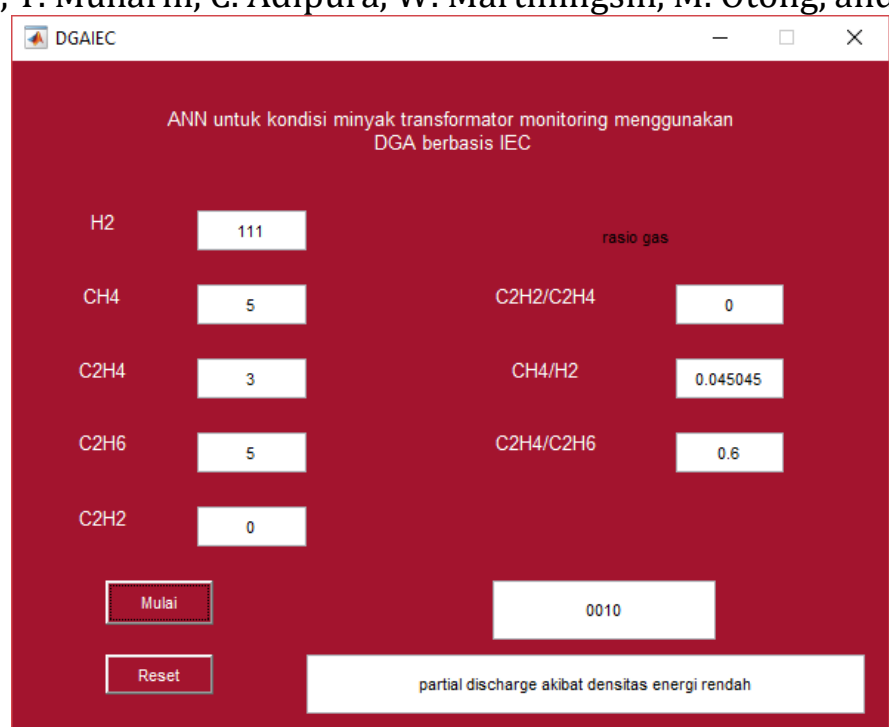

Figure 2: GUI Panel

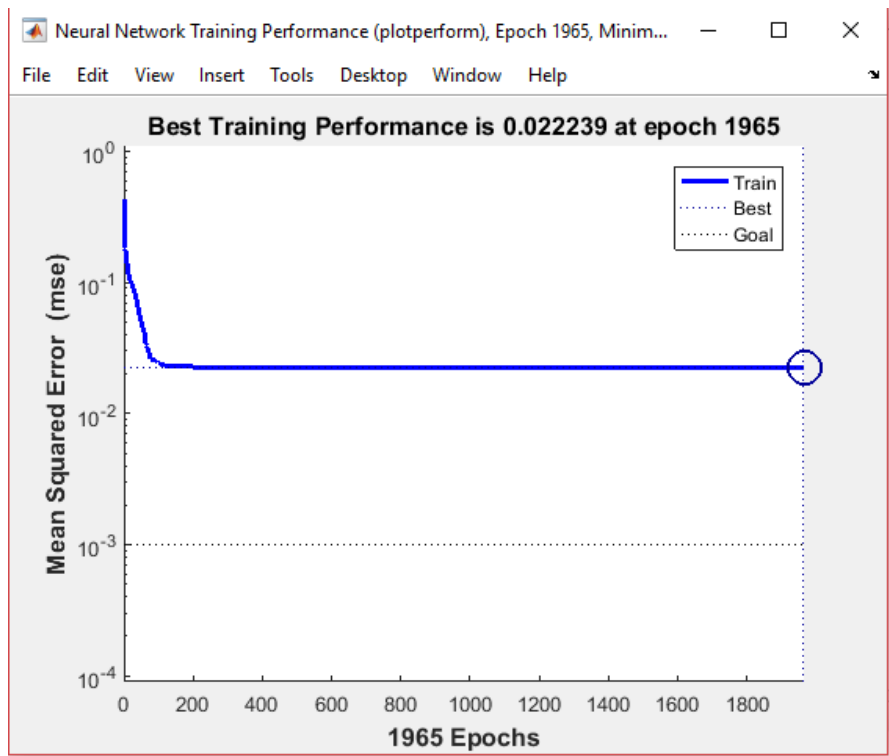

Figure 3: Training Performance

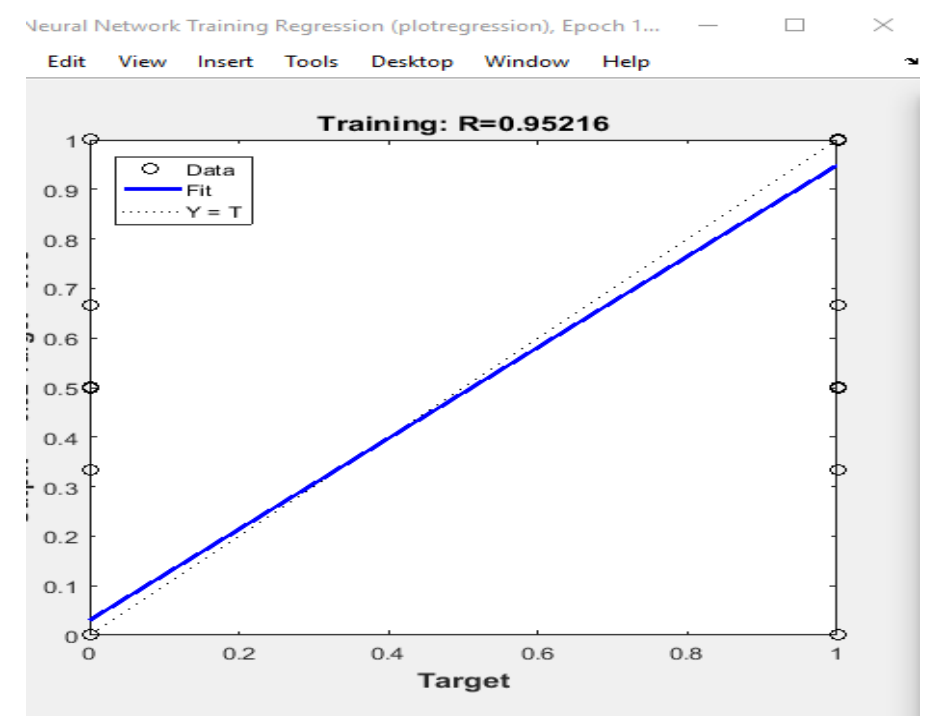

Figure 4: Regression 
Analysis of Power Transformator Conditions Using DGA Method Using Artificial Neural Network in Krakatau Electrical Power Company

Regression in the preprocessing process, on targets with network output values $0-1$. In this regression plot shows the relationship between the actual data and the output data from the Artificial Neural Network on the training data. The coefficient $\mathrm{R}$ is 0.95216 close to 1 , showing good results for the compatibility of the output with the actual data. For general error analysis purposes, all errors are categorized into nine error codes. Codes 0001 through 1001 are assigned to this error as shown in the table.

Table 6: Initialize DGA output ANN

\begin{tabular}{|l|c|}
\hline Type of Disturbance & Nilai Output \\
\hline Normal (N) & 0001 \\
\hline PD due to low energy density & 0010 \\
\hline PD due to High energy density & 0011 \\
\hline D1 & 0100 \\
\hline D2 & 0101 \\
\hline TR $<150^{\circ} \mathrm{C}$ & 0110 \\
\hline TR Between $150^{\circ} \mathrm{C}-300^{\circ} \mathrm{C}$ & 0111 \\
\hline TR Between $300^{\circ} \mathrm{C}-700^{\circ} \mathrm{C}$ & 1000 \\
\hline TT $>700^{\circ} \mathrm{C}$ & 1001 \\
\hline
\end{tabular}

The desired result in this design is to be able to know the gas fault that occurs in the transformer oil and can make it easier to analyze faults based on the gas content in the transformer oil. The table is a comparison of gas data for parameters R1, R2, R3 that are used to test artificial neural networks based on conditions. It can be seen that in each condition it represents a cross fault.

\begin{tabular}{|c|c|c|c|c|c|c|c|c|c|}
\hline \multirow{2}{*}{ NO } & \multicolumn{3}{|c|}{ Parameter Input } & \multicolumn{4}{|c|}{ Parameter Output } & \multirow{2}{*}{ Code } & \multirow{2}{*}{ KONDISI } \\
\hline & $\mathrm{xl}$ & $\mathrm{x} 2$ & $\mathrm{x} 3$ & $\mathrm{yl}$ & $\mathrm{y} 2$ & $\mathrm{y} 3$ & y4 & & \\
\hline 1 & 0 & 0.428571 & 0.555556 & 0 & 0 & 0 & 1 & $\mathrm{Fl}$ & Normal \\
\hline 2 & 0 & 1 & 0.285714 & 0 & 0 & 0 & 1 & $\mathrm{Fl}$ & Normal \\
\hline 3 & 0 & 0.666667 & 1.4 & 0 & 1 & 1 & 0 & F6 & Termal rendah $<150^{\circ} \mathrm{C}$ \\
\hline 4 & 0 & 0.045045 & 0.6 & 0 & 0 & 1 & 0 & F2 & Partial discharge akibat densitas energi rendah \\
\hline 5 & 0 & 0.25 & 0.5 & 0 & 0 & 0 & 1 & $\mathrm{Fl}$ & Normal \\
\hline 6 & 0.75 & 1.333333 & 0.666667 & 0 & 0 & 0 & 1 & $\mathrm{Fl}$ & Normal \\
\hline 7 & 0 & 0.6 & 0.75 & 0 & 0 & 0 & 1 & $\mathrm{Fl}$ & Normal \\
\hline 8 & 0.45 & 1.111111 & 0.333333 & 0 & 0 & 0 & 1 & $\mathrm{Fl}$ & Normal \\
\hline 9 & 0 & 0.428571 & 4 & 0 & 0 & 0 & 1 & $\mathrm{Fl}$ & Normal \\
\hline 10 & 0 & 0.5 & 0.2 & 0 & 0 & 0 & 1 & $\mathrm{Fl}$ & Normal \\
\hline 11 & 0 & 1 & 1.666667 & 0 & 1 & 1 & 0 & F6 & Termal rendah $<150^{\circ} \mathrm{C}$ \\
\hline 12 & 0 & 0.4 & 0.333333 & 0 & 0 & 0 & 1 & $\mathrm{Fl}$ & Normal \\
\hline 13 & 0 & 0.5 & 0.333333 & 0 & 0 & 0 & 1 & $\mathrm{Fl}$ & Normal \\
\hline 14 & 0 & 0.333333 & 0.142857 & 0 & 0 & 0 & 1 & $\mathrm{Fl}$ & Normal \\
\hline 15 & 0 & 0.625 & 0.071429 & 0 & 0 & 0 & 1 & $\mathrm{Fl}$ & Normal \\
\hline 16 & 0 & 0.666667 & 1 & 0 & 0 & 0 & 1 & $\mathrm{Fl}$ & Normal \\
\hline 17 & 0 & 0.181818 & 0.8 & 0 & 0 & 0 & 1 & $\mathrm{Fl}$ & Normal \\
\hline 18 & 0.1125 & 0.263158 & 1.333333 & 0 & 1 & 0 & 0 & $\mathrm{~F} 4$ & Discharge energi rendah \\
\hline 19 & 0 & 0.117647 & 0.6 & 0 & 0 & 0 & 1 & $\mathrm{Fl}$ & Normal \\
\hline
\end{tabular}

Figure 5: Test data in Parameters

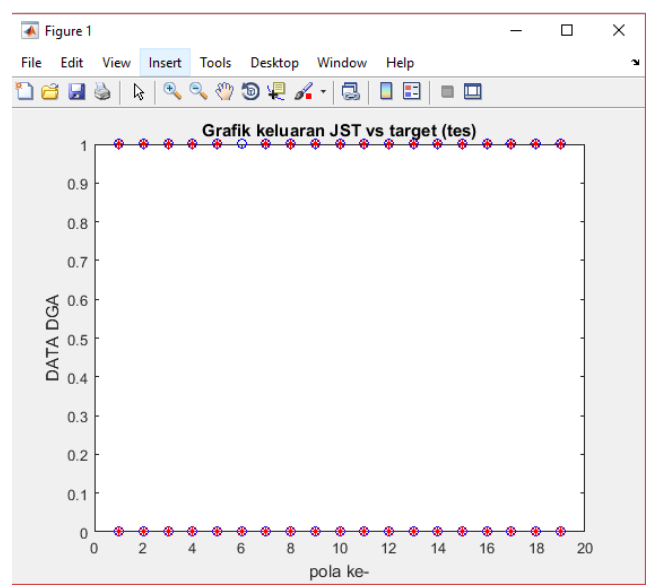

Figure 6: Comparison of JST with Actual 
Hartono, Y. Muharni, C. Adipura, W. Martiningsih, M. Otong, and M. Irvan

Is the comparison of target data with network output. Comparison between targets and ANN output can be seen in the table.

Table 7: Comparison Between Target Data and Output Data JST

\begin{tabular}{|l|c|c|}
\hline No & Target Data & Data Output JST \\
\hline 1 & 0001 & 0001 \\
\hline 2 & 0001 & 0001 \\
\hline 3 & 0110 & 0110 \\
\hline 4 & 0010 & 0010 \\
\hline 5 & 0001 & 0001 \\
\hline 6 & 0001 & 0100 \\
\hline 7 & 0001 & 0001 \\
\hline 8 & 0001 & 0001 \\
\hline 9 & 0001 & 0001 \\
\hline 10 & 0001 & 0001 \\
\hline 11 & 0110 & 0110 \\
\hline 12 & 0001 & 0001 \\
\hline 13 & 0001 & 0001 \\
\hline 14 & 0001 & 0001 \\
\hline 15 & 0001 & 0001 \\
\hline 16 & 0001 & 0001 \\
\hline 17 & 0001 & 0001 \\
\hline 18 & 0100 & 0100 \\
\hline 19 & 0001 & 0001 \\
\hline
\end{tabular}

To find out how valid the results of the test can use the formula level of accuracy.

Level of Accuracy $(\%)=\frac{\text { test data is corret }}{\text { amount of test data }} \times 100 \%$

Level of Accuracy(\%) $=\frac{18}{19} \times 100 \%$

Level of Accuracy(\%) $=0,947 \times 100 \%$

Level of Accuracy $(\%)=94.7 \%$.

\section{CONCLUSIONS \& RECOMMENDATIONS}

From the research that has been done can be concluded among other things:

Based on the conclusion of the experimental results, the artificial neural network model with the 3 hidden layer network architecture is the most optimal, in the first hidden layer, 10 neurons are arranged and the second and third are 20 neurons using the transfer logic function, and the output layer 4 neurons with the logic activation function. So that it has a correlation coefficient (regression) of 0.95216 and MSE (Mean Square Error) is worth 0.000216. the accuracy obtained is $94.4 \%$. 


\section{Analysis of Power Transformator Conditions Using DGA Method Using Artificial Neural Network in Krakatau Electrical Power Company}

\section{APPENDICES}

\begin{tabular}{|c|c|c|c|c|c|c|c|c|c|c|c|c|c|c|c|}
\hline \multirow{2}{*}{ no } & \multicolumn{3}{|c|}{ Input } & \multicolumn{4}{|c|}{ Target } & \multirow{2}{*}{ no } & \multicolumn{3}{|c|}{ Input } & \multicolumn{4}{|c|}{ Target } \\
\hline & $\mathrm{x1}$ & $x 2$ & $x 3$ & $\mathrm{y} 1$ & $y 2$ & y3 & $\mathrm{y} 4$ & & $x 1$ & $x 2$ & $x 3$ & y1 & $y^{2}$ & y3 & $\mathrm{y}^{4}$ \\
\hline 1 & 0 & 0.625 & 0.3 & 0 & 0 & 0 & 1 & 26 & 0 & \begin{tabular}{|l|l}
0.333333 \\
\end{tabular} & 0.857143 & 0 & 0 & 0 & 1 \\
\hline 2 & 0 & 1 & 0.12 & 0 & 0 & 0 & 1 & 27 & 0 & \begin{tabular}{|l|}
0.352941 \\
\end{tabular} & 0.625 & 0 & 0 & 0 & 1 \\
\hline 3 & 0 & 0.888889 & 0.285714 & 0 & 0 & 0 & 1 & 28 & 0.5 & 0.25 & \begin{tabular}{|l|}
0.333333 \\
\end{tabular} & 0 & 0 & 0 & 1 \\
\hline 4 & 0 & 0.166667 & 0.444444 & 0 & 0 & 0 & 1 & 29 & 0.5 & 0.2 & 0.333333 & 0 & 0 & 0 & 1 \\
\hline 5 & 0 & 0.826087 & 0.066667 & 0 & 0 & 0 & 1 & 30 & 0 & 0.4 & 0.8 & 0 & 0 & 0 & 1 \\
\hline 6 & 0 & 0.8 & 0.833333 & 0 & 0 & 0 & 1 & 31 & 0 & 0.666667 & 0.2 & 0 & 0 & 0 & 1 \\
\hline 7 & 1 & 0.8 & 0.125 & 0 & 0 & 0 & 1 & 32 & 0 & \begin{tabular}{|l}
0.333333 \\
\end{tabular} & 0.6 & 0 & 0 & 0 & 1 \\
\hline 8 & 1 & 3.363636 & 0.166667 & 0 & 0 & 0 & 1 & 33 & 0 & 0.75 & 0.2 & 0 & 0 & 0 & 1 \\
\hline 9 & 0 & 0.111111 & 1 & 0 & 0 & 0 & 1 & 34 & 0.25 & 0.4 & \begin{tabular}{|l|l|}
0.666667 \\
\end{tabular} & 0 & 0 & 0 & 1 \\
\hline 10 & 0 & 0.4 & 0.75 & 0 & 0 & 0 & 1 & 35 & 0 & 0.4 & 0.8 & 0 & 0 & 0 & 1 \\
\hline 11 & 0 & 0.8 & 0.571429 & 0 & 0 & 0 & 1 & 36 & 0 & \begin{tabular}{|l|}
0.666667 \\
\end{tabular} & 0.285714 & 0 & 0 & 0 & 1 \\
\hline 12 & 0.166667 & 0.6 & 0.6 & 0 & 0 & 0 & 1 & 37 & 0.25 & 0.8 & \begin{tabular}{|l|l|}
0666667 \\
\end{tabular} & 0 & 0 & 0 & 1 \\
\hline 13 & 0 & 0.4 & 0.6 & 0 & 0 & 0 & 1 & 38 & 0 & 0.4 & 0.75 & 0 & 0 & 0 & 1 \\
\hline 14 & 0.5 & 1.2 & \begin{tabular}{|l|}
0.166667 \\
\end{tabular} & 0 & 0 & 0 & 1 & 39 & 0.487097 & 4.8 & 5.166667 & 0 & 0 & 0 & 1 \\
\hline 15 & 0 & 0.6 & 0.5 & 0 & 0 & 0 & 1 & 40 & 0.665 & 2.4 & 4 & 0 & 0 & 0 & 1 \\
\hline 16 & 0 & 0.8 & \begin{tabular}{|l|l}
0.142857 \\
\end{tabular} & 0 & 0 & 0 & 1 & 41 & 0.611765 & 1.6 & 4.25 & 0 & 0 & 0 & 1 \\
\hline 17 & 0 & 0.384615 & 0.25 & 0 & 0 & 0 & 1 & 42 & 0.413043 & 1.125 & 4.6 & 0 & 0 & 0 & 1 \\
\hline 18 & 0 & 0.8 & 0.142857 & 0 & 0 & 0 & 1 & 43 & 0.37027 & 1.272727 & \begin{tabular}{|l}
5.285714 \\
\end{tabular} & 0 & 0 & 0 & 1 \\
\hline 19 & 0.5 & 0.4 & 0.2 & 0 & 0 & 0 & 1 & 44 & 0.483333 & 2.4 & 5 & 0 & 0 & 0 & 1 \\
\hline 20 & 0 & 0.666667 & 0.5 & 0 & 0 & 0 & 1 & 45 & 1 & 6.666667 & 4 & 0 & 0 & 0 & 1 \\
\hline 21 & 0 & 0.4 & 0.333333 & 0 & 0 & 0 & 1 & 46 & 0 & 0.568182 & 0.155844 & 0 & 0 & 0 & 1 \\
\hline 22 & 0 & 0.4 & 0.75 & 0 & 0 & 0 & 1 & 47 & 2 & 0.239726 & 0.333333 & 0 & 0 & 0 & 1 \\
\hline 23 & 0.125 & 0.857143 & 0.8 & 0 & 0 & 0 & 1 & 48 & 0.125 & 0.166667 & 0.222222 & 0 & 0 & 0 & 1 \\
\hline 24 & 0.666667 & \begin{tabular}{|l}
1.333333 \\
\end{tabular} & 1.5 & 0 & 0 & 0 & 1 & 49 & 0 & 0.285714 & \begin{tabular}{|l|}
0.1111111 \\
\end{tabular} & 0 & 0 & 0 & 1 \\
\hline 25 & 0 & 0.375 & 0.8 & 0 & 0 & 0 & 1 & 50 & 0.25 & 0.358209 & 0.125 & 0 & 0 & 0 & 1 \\
\hline
\end{tabular}

\begin{tabular}{|c|c|c|c|c|c|c|c|c|c|c|c|c|c|c|c|}
\hline \multirow{2}{*}{ no } & \multicolumn{3}{|c|}{ Input } & \multicolumn{4}{|c|}{ Target } & \multirow{2}{*}{ no } & \multicolumn{3}{|c|}{ Input } & \multicolumn{4}{|c|}{ Target } \\
\hline & $\mathrm{x} 1$ & $x 2$ & $x 3$ & y1 & $y 2$ & y3 & y4 & & $\mathrm{x} 1$ & $x 2$ & $x 3$ & y1 & $y 2$ & y3 & y4 \\
\hline 51 & 0 & 0.42029 & 0.121951 & 0 & 0 & 0 & 1 & 76 & 0.026316 & 0.571429 & \begin{tabular}{|l|l|}
0.260274 \\
\end{tabular} & 0 & 0 & 0 & 1 \\
\hline 52 & 0 & 0.191489 & 0.122807 & 0 & 0 & 0 & 1 & 77 & 0 & 0.533333 & 0.666667 & 0 & 0 & 0 & 1 \\
\hline 53 & 0.064286 & 0.10084 & 0.112903 & 0 & 0 & 0 & 1 & 78 & 0 & 1 & 0.571429 & 0 & 0 & 0 & 1 \\
\hline 54 & 2 & 0.239726 & \begin{tabular}{|l|}
0.333333 \\
\end{tabular} & 0 & 0 & 0 & 1 & 79 & 0.5 & 1.571429 & 0.125 & 0 & 0 & 0 & 1 \\
\hline 55 & 0.083333 & 0.206704 & 0.428571 & 0 & 0 & 0 & 1 & 80 & 0.142857 & 0.176471 & 0.7 & 0 & 0 & 0 & 1 \\
\hline 56 & 0.09 & 0.266667 & 0.833333 & 0 & 0 & 0 & 1 & 81 & 0.071429 & 0.197674 & 1 & 0 & 0 & 0 & 1 \\
\hline 57 & 1 & 0.5 & 0.666667 & 0 & 0 & 0 & 1 & 82 & 0 & 0.5 & 1 & 0 & 0 & 0 & 1 \\
\hline 58 & 0.05 & 0.3375 & \begin{tabular}{|l|}
0.16129 \\
\end{tabular} & 0 & 0 & 0 & 1 & 83 & 0 & 0.727273 & 0.2 & 0 & 0 & 0 & 1 \\
\hline 59 & 0 & 0.875 & 0.2 & 0 & 0 & 0 & 1 & 84 & 0 & 1 & 0.083333 & 0 & 0 & 0 & 1 \\
\hline 60 & 0.45 & 0.666667 & 0.333333 & 0 & 0 & 0 & 1 & 85 & 0 & 0.75 & \begin{tabular}{|l|l}
0.777778 \\
\end{tabular} & 0 & 0 & 0 & 1 \\
\hline 61 & 0.25 & 1.428571 & 0.666667 & 0 & 0 & 0 & 1 & 86 & 2.277327 & 0.068764 & 139.6667 & 0 & 0 & 0 & 1 \\
\hline 62 & 0.4 & 0.615385 & 0.166667 & 0 & 0 & 0 & 1 & 87 & 0.026316 & 0.8 & 0.260274 & 0 & 0 & 0 & 1 \\
\hline 63 & 0 & 0.465517 & 0.315789 & 0 & 0 & 0 & 1 & 88 & 0.166667 & 3.8 & 2 & 0 & 0 & 0 & 1 \\
\hline 64 & 0 & 0.47619 & 0.333333 & 0 & 0 & 0 & 1 & 89 & 1.5 & 4.2 & 0.333333 & 0 & 0 & 0 & 1 \\
\hline 65 & 0 & 0.416667 & 0.25 & 0 & 0 & 0 & 1 & 90 & 0 & 0.189655 & 0.153846 & 0 & 0 & 0 & 1 \\
\hline 66 & 0.1 & 0.483871 & 0.454545 & 0 & 0 & 0 & 1 & 91 & 0.142857 & 2.4 & 1.75 & 0 & 0 & 0 & 1 \\
\hline 67 & 0 & 0.45 & 0.333333 & 0 & 0 & 0 & 1 & 92 & 0.0625 & 0.8 & 1 & 0 & 0 & 0 & 1 \\
\hline 68 & 0.291026 & 1.128205 & 11.14286 & 0 & 0 & 0 & 1 & 93 & 0.25 & 1.6 & 1 & 0 & 0 & 0 & 1 \\
\hline 69 & 15 & 0.296296 & 0.05 & 0 & 0 & 0 & 1 & 94 & 0 & 0.2 & 0.8 & 0 & 0 & 0 & 1 \\
\hline 70 & 0 & 0.2 & 0.8 & 0 & 0 & 0 & 1 & 95 & 0 & 0.666667 & 0.166667 & 0 & 0 & 0 & 1 \\
\hline 71 & 0 & 0.189655 & 0.153846 & 0 & 0 & 0 & 1 & 96 & 0.25 & 0.833333 & 0.333333 & 0 & 0 & 0 & 1 \\
\hline 72 & 0 & 0.666667 & 0.166667 & 0 & 0 & 0 & 1 & 97 & 0.5 & 1 & 0.083333 & 0 & 0 & 0 & 1 \\
\hline 73 & 0.25 & 0.833333 & 0.333333 & 0 & 0 & 0 & 1 & 98 & 0 & 1 & 1 & 0 & 0 & 0 & 1 \\
\hline 74 & 0.142857 & 2.4 & 1.75 & 0 & 0 & 0 & 1 & 99 & 0 & 0.727273 & 0.666667 & 0 & 0 & 0 & 1 \\
\hline 75 & 0.125 & 1 & 0.444444 & 0 & 0 & 0 & 1 & 100 & 0 & 0.727273 & 0.2 & 0 & 0 & 0 & 1 \\
\hline
\end{tabular}


Hartono, Y. Muharni, C. Adipura, W. Martiningsih, M. Otong, and M. Irvan

\begin{tabular}{|c|c|c|c|c|c|c|c|c|c|c|c|c|c|c|c|}
\hline \multirow{2}{*}{ no } & \multicolumn{3}{|c|}{ Input } & \multicolumn{4}{|c|}{ Target } & \multirow{2}{*}{ no } & \multicolumn{3}{|c|}{ Input } & \multicolumn{4}{|c|}{ Target } \\
\hline & $\mathrm{x} 1$ & $x 2$ & $x 3$ & $\mathrm{y} 1$ & $y 2$ & y3 & y4 & & $\mathrm{x} 1$ & $x 2$ & $x 3$ & y1 & y2 & y3 & $\mathrm{y} 4$ \\
\hline 101 & 0 & 1 & 0.083333 & 0 & 0 & 0 & 1 & 126 & 1 & 0.8 & 0.125 & 0 & 1 & 0 & 0 \\
\hline 102 & 0 & 0.5 & 1 & 0 & 0 & 0 & 1 & 127 & 0.25 & 0.4 & 2 & 0 & 1 & 0 & 0 \\
\hline 103 & 0 & 0.75 & 0.777778 & 0 & 0 & 0 & 1 & 128 & 0.166667 & 0.5 & 0.6 & 0 & 1 & 0 & 0 \\
\hline 104 & 0 & 0.578947 & 0.791667 & 0 & 0 & 0 & 1 & 129 & 0.375 & 1 & 2 & 0 & 1 & 0 & 0 \\
\hline 105 & 0 & 0.714286 & 0.05 & 0 & 0 & 0 & 1 & 130 & 0.3 & 1.333333 & 1.25 & 0 & 1 & 0 & 0 \\
\hline 106 & 0 & 0.769231 & 0.125 & 0 & 0 & 0 & 1 & 131 & 0.5 & 0.4 & 2 & 0 & 1 & 0 & 0 \\
\hline 107 & 0 & \begin{tabular}{|l|l|}
0.87234 \\
\end{tabular} & 0.285714 & 0 & 0 & 0 & 1 & 132 & \begin{tabular}{|l|}
0.16129 \\
\end{tabular} & 1.222222 & 1.24 & 0 & 1 & 0 & 0 \\
\hline 108 & 0.291026 & 1.128205 & 11.14286 & 0 & 0 & 0 & 1 & 133 & 0.155556 & 1.066667 & 1.25 & 0 & 1 & 0 & 0 \\
\hline 109 & 0.05 & 0.3375 & 0.16129 & 0 & 0 & 0 & 1 & 134 & 0.217391 & 0.695652 & 1.121951 & 0 & 1 & 0 & 0 \\
\hline 110 & 0.125 & 1.04 & 0.070175 & 0 & 0 & 0 & 1 & 135 & 0.680556 & 0.309735 & 1.531915 & 0 & 1 & 0 & 0 \\
\hline 111 & 0.083333 & 0.919355 & 0.098361 & 0 & 0 & 0 & 1 & 136 & 0.689231 & 0.368687 & 1.666667 & 0 & 1 & 0 & 0 \\
\hline 112 & 0.375 & 2.411765 & 4 & 0 & 0 & 0 & 1 & 137 & 0.144231 & 0.66129 & 1.333333 & 0 & 1 & 0 & 0 \\
\hline 113 & 0.055556 & 0.426471 & 0.134328 & 0 & 0 & 0 & 1 & 138 & 0.5 & 0.4 & 2 & 0 & 1 & 0 & 0 \\
\hline 114 & 0.071429 & 0.014423 & 0.170732 & 0 & 0 & 1 & 0 & 139 & 11.3 & 0.352113 & 1 & 0 & 1 & 0 & 0 \\
\hline 115 & 0.010477 & 0.111804 & 5.688742 & 0 & 0 & 1 & 0 & 140 & 4.8 & 0.408163 & 1.5 & 0 & 1 & 0 & 0 \\
\hline 116 & 0.010417 & 0.080255 & 5.7 & 0 & 0 & 1 & 0 & 141 & 0.166667 & 0.2 & 0.6 & 0 & 1 & 0 & 0 \\
\hline 117 & 0.008436 & 0.074151 & 5.521739 & 0 & 0 & 1 & 0 & 142 & 0.214286 & 0.193182 & 1.166667 & 0 & 1 & 0 & 0 \\
\hline 118 & 0.010417 & 0.080255 & 5.7 & 0 & 0 & 1 & 0 & 143 & 0.666667 & 0.238095 & 0.6 & 0 & 1 & 0 & 0 \\
\hline 119 & 0.125 & 0.714286 & 0.444444 & 0 & 0 & 1 & 0 & 144 & 0.5 & 0.2 & 0.25 & 0 & 1 & 0 & 0 \\
\hline 120 & 0.071429 & 0.014423 & 0.170732 & 0 & 0 & 1 & 0 & 145 & 2.930769 & 0.175758 & 2.6 & 0 & 1 & 0 & 0 \\
\hline 121 & 0.010791 & 0.149778 & 5.813665 & 0 & 0 & 1 & 0 & 146 & 1.833333 & 0.571429 & 3 & 0 & 1 & 0 & 0 \\
\hline 122 & 0 & 0.089744 & 0.583333 & 0 & 0 & 1 & 0 & 147 & 0.5 & 0.202128 & 1 & 0 & 1 & 0 & 0 \\
\hline 123 & 1.25 & 0.065217 & 0.8 & 0 & 0 & 1 & 1 & 148 & 2.930769 & 0.175758 & 2.6 & 0 & 1 & 0 & 0 \\
\hline 124 & 0.833333 & 0.1 & 0.75 & 0 & 0 & 1 & 1 & 149 & 0.5 & 0.28 & 1.8 & 0 & 1 & 0 & 0 \\
\hline 125 & 0.1 & 0.5 & 5 & 0 & 1 & 0 & 0 & 150 & 0.222222 & 0.235294 & 1.285714 & 0 & 1 & 0 & 0 \\
\hline
\end{tabular}

\begin{tabular}{|c|c|c|c|c|c|c|c|c|c|c|c|c|c|c|c|}
\hline \multirow{2}{*}{ no } & \multicolumn{3}{|c|}{ Input } & \multicolumn{4}{|c|}{ Target } & \multirow{2}{*}{ no } & \multicolumn{3}{|c|}{ Input } & \multicolumn{4}{|c|}{ Target } \\
\hline & $x 1$ & $x 2$ & $x 3$ & $\mathrm{y} 1$ & y2 & y3 & y4 & & $x 1$ & $x 2$ & $x 3$ & $y 1$ & $y 2$ & y3 & $\mathrm{y} 4$ \\
\hline 151 & 0.7 & 1.8 & 1.25 & 0 & 1 & 0 & 0 & 176 & 2.333333 & 0.444444 & 0.6 & 0 & 1 & 0 & 0 \\
\hline 152 & 0.1875 & 0.214286 & 1.142857 & 0 & 1 & 0 & 0 & 177 & 0.833333 & 0.454545 & 0.5 & 0 & 1 & 0 & 0 \\
\hline 153 & 0.680556 & 0.309735 & 1.531915 & 0 & 1 & 0 & 0 & 178 & 0.6 & 1 & 1.666667 & 0 & 1 & 0 & 0 \\
\hline 154 & 0.222222 & 0.235294 & 1.285714 & 0 & 1 & 0 & 0 & 179 & 0.333333 & 0.714286 & 0.75 & 0 & 1 & 0 & 0 \\
\hline 155 & 4.626316 & 0.34375 & 12.66667 & 0 & 1 & 0 & 0 & 180 & 0.125 & 0.2 & 2 & 0 & 1 & 0 & 0 \\
\hline 156 & 4.968 & 0.270833 & 8.333333 & 0 & 1 & 0 & 0 & 181 & 1.00625 & 0.141176 & 2.666667 & 0 & 1 & 0 & 0 \\
\hline 157 & 15.25 & 0.179104 & 8 & 0 & 1 & 0 & 0 & 182 & 0.916667 & 2.375 & 2.181818 & 0 & 1 & 0 & 0 \\
\hline 158 & 7.06 & 0.163265 & 5 & 0 & 1 & 0 & 0 & 183 & 0.5 & 0.875 & 1 & 0 & 1 & 0 & 0 \\
\hline 159 & \begin{tabular}{|l|}
9.030769 \\
\end{tabular} & 0.147541 & 6.5 & 0 & 1 & 0 & 0 & 184 & 1.5111111 & 0.823529 & 2 & 0 & 1 & 0 & 0 \\
\hline 160 & 17.62 & 0.241379 & 2.5 & 0 & 1 & 0 & 0 & 185 & 0.1 & 0.888889 & 2.5 & 0 & 1 & 0 & 0 \\
\hline 161 & 10.3 & 0.222222 & 0.2 & 0 & 1 & 0 & 0 & 186 & 0.1 & 0.888889 & 2.5 & 0 & 1 & 0 & 0 \\
\hline 162 & 19.84 & 0.083333 & 5 & 0 & 1 & 0 & 0 & 187 & 0.5 & 0.4 & 1 & 0 & 1 & 0 & 0 \\
\hline 163 & 6.72 & 0.156863 & 3.75 & 0 & 1 & 0 & 0 & 188 & 1.754286 & 0.008667 & 5.833333 & 0 & 1 & 0 & 0 \\
\hline 164 & 0.125 & 0.5 & 1.333333 & 0 & 1 & 0 & 0 & 189 & 0.25 & 0.428571 & 1.333333 & 0 & 1 & 0 & 0 \\
\hline 165 & 0.166667 & 0.1875 & 0.428571 & 0 & 1 & 0 & 0 & 190 & 0.166667 & 0.2 & 1.5 & 0 & 1 & 0 & 0 \\
\hline 166 & 0.1 & \begin{tabular}{|c|}
0.333333 \\
\end{tabular} & 0.714286 & 0 & 1 & 0 & 0 & 191 & 1.9 & 0.109375 & 1.666667 & 0 & 1 & 0 & 0 \\
\hline 167 & 0.5 & 0.4375 & 0.2 & 0 & 1 & 0 & 0 & 192 & 0.071429 & 0.269231 & 3.5 & 0 & 1 & 0 & 0 \\
\hline 168 & 0.125 & 0.5 & 1.333333 & 0 & 1 & 0 & 0 & 193 & 0.125 & 0.285714 & 4 & 0 & 1 & 0 & 1 \\
\hline 169 & 0.166667 & 0.1875 & 0.428571 & 0 & 1 & 0 & 0 & 194 & 2.94 & 0.22561 & 10 & 0 & 1 & 0 & 1 \\
\hline 170 & 0.1 & 0.333333 & 0.714286 & 0 & 1 & 0 & 0 & 195 & \begin{tabular}{|l|}
1.916667 \\
\end{tabular} & 0.235294 & 6 & 0 & 1 & 0 & 1 \\
\hline 171 & 0.5 & 0.285714 & 0.5 & 0 & 1 & 0 & 0 & 196 & 0.5867 & 0.211938 & 21.9337 & 0 & 1 & 0 & 1 \\
\hline 172 & 0.375 & 0.25 & 0.666667 & 0 & 1 & 0 & 0 & 197 & 2.321875 & 0.666667 & 32 & 0 & 1 & 0 & 1 \\
\hline 173 & 0.25 & 0.142857 & 1.6 & 0 & 1 & 0 & 0 & 198 & 0 & 0.4 & 3.5 & 0 & 1 & 0 & 1 \\
\hline 174 & 3.983333 & 0.2 & 1 & 0 & 1 & 0 & 0 & 199 & 1.673913 & 0.692308 & 3.285714 & 0 & 1 & 0 & 1 \\
\hline 175 & 0.75 & 0.909091 & 1.142857 & 0 & 1 & 0 & 0 & 200 & 0.125 & 0.6 & 4 & 0 & 1 & 0 & 1 \\
\hline
\end{tabular}


Analysis of Power Transformator Conditions Using DGA Method Using Artificial Neural Network in Krakatau Electrical Power Company

\begin{tabular}{|c|c|c|c|c|c|c|c|c|c|c|c|c|c|c|c|}
\hline \multirow{2}{*}{ no } & \multicolumn{3}{|c|}{ Input } & \multicolumn{4}{|c|}{ Target } & \multirow{2}{*}{ no } & \multicolumn{3}{|c|}{ Input } & \multicolumn{4}{|c|}{ Target } \\
\hline & $x 1$ & $\times 2$ & x3 & $\mathrm{y} 1$ & $y^{2}$ & y3 & y4 & & $x 1$ & $x 2$ & $x 3$ & $\mathrm{y} 1$ & $y^{2}$ & $\mathrm{y}^{3}$ & y4 \\
\hline 201 & 1.921739 & 0.846154 & 3.285714 & 0 & 1 & 0 & 1 & 226 & 0.010204 & 0.733333 & 1.088889 & 0 & 1 & 1 & 0 \\
\hline 202 & 1.673913 & 0.692308 & 3.285714 & 0 & 1 & 0 & 1 & 227 & 0.010638 & 0.75 & 1.424242 & 0 & 1 & 1 & 0 \\
\hline 203 & 15 & 0.296296 & 0.05 & 0 & 1 & 0 & 1 & 228 & \begin{tabular}{|l|}
0.011364 \\
\end{tabular} & 0.8 & 1.257143 & 0 & 1 & 1 & 0 \\
\hline 204 & 2.919048 & 0.172297 & 63 & 0 & 1 & 0 & 1 & 229 & 0.015789 & 0.56 & 2.375 & 0 & 1 & 1 & 0 \\
\hline 205 & 1.921739 & 0.846154 & 3.285714 & 0 & 1 & 0 & 1 & 230 & 0.1 & 0.333333 & 0.714286 & 0 & 1 & 1 & 0 \\
\hline 206 & 1.673913 & 0.692308 & 3.285714 & 0 & 1 & 0 & 1 & 231 & 0.5 & 0.4375 & 0.2 & 0 & 1 & 1 & 0 \\
\hline 207 & 0.279259 & 0.98913 & 7.105263 & 0 & 1 & 0 & 1 & 232 & 0.016667 & 0.5 & 3 & 0 & 1 & 1 & 0 \\
\hline 208 & 0.342063 & 0.728972 & 7.411765 & 0 & 1 & 0 & 1 & 233 & 0.0625 & 0.571429 & 1 & 0 & 1 & 1 & 0 \\
\hline 209 & 0.42029 & 0.560241 & 9.2 & 0 & 1 & 0 & 1 & 234 & 0.090909 & 0.325 & 1.571429 & 0 & 1 & 1 & 0 \\
\hline 210 & 0.342063 & 0.728972 & 7.411765 & 0 & 1 & 0 & 1 & 235 & 0.0625 & 0.8 & 1 & 0 & 1 & 1 & 0 \\
\hline 211 & 0.279259 & \begin{tabular}{|l|}
0.98913 \\
\end{tabular} & 7.105263 & 0 & 1 & 0 & 1 & 236 & 0.25 & 1.6 & 1 & 0 & 1 & 1 & 0 \\
\hline 212 & 2.05 & 0.010959 & 6.5 & 0 & 1 & 0 & 1 & 237 & 0.055556 & \begin{tabular}{|l|}
0.857143 \\
\end{tabular} & \begin{tabular}{|l}
1.285714 \\
\end{tabular} & 0 & 1 & 1 & 0 \\
\hline 213 & 2.357143 & 0.029909 & 7 & 0 & 1 & 0 & 1 & 238 & 0 & 0.551724 & 1.7 & 0 & 1 & 1 & 0 \\
\hline 214 & 0.125 & 0.714286 & 0.444444 & 0 & 1 & 0 & 1 & 239 & 0 & 0.515152 & 1.75 & 0 & 1 & 1 & 0 \\
\hline 215 & 1.673913 & 0.692308 & 3.285714 & 0 & 1 & 0 & 1 & 240 & 0 & 0.5 & 1.125 & 0 & 1 & 1 & 0 \\
\hline 216 & 0 & 0.727273 & 1.75 & 0 & 1 & 1 & 0 & 241 & 0.090909 & 0.325 & 1.571429 & 0 & 1 & 1 & 0 \\
\hline 217 & 0 & 1 & 2 & 0 & 1 & 1 & 0 & 242 & 0.055556 & 0.857143 & 1.285714 & 0 & 1 & 1 & 0 \\
\hline 218 & 0 & 0.545455 & 1.25 & 0 & 1 & 1 & 0 & 243 & \begin{tabular}{|l|}
0.142857 \\
\end{tabular} & 0.176471 & 0.7 & 0 & 1 & 1 & 0 \\
\hline 219 & 0 & 1 & 1.333333 & 0 & 1 & 1 & 0 & 244 & 0.071429 & 0.197674 & 1 & 0 & 1 & 1 & 0 \\
\hline 220 & 0.25 & 2.5 & 0.166667 & 0 & 1 & 1 & 0 & 245 & 0 & 1 & 0.571429 & 0 & 1 & 1 & 0 \\
\hline 221 & 0.15 & 0.666667 & 0.75 & 0 & 1 & 1 & 0 & 246 & 0.5 & 1.571429 & 0.125 & 0 & 1 & 1 & 0 \\
\hline 222 & 0 & 1 & 2 & 0 & 1 & 1 & 0 & 247 & 0 & \begin{tabular}{|l|l|}
0.551724 \\
\end{tabular} & 1.7 & 0 & 1 & 1 & 0 \\
\hline 223 & \begin{tabular}{|l|}
0.00641 \\
\end{tabular} & 0.619048 & 2.052632 & 0 & 1 & 1 & 0 & 248 & 0 & 0.515152 & 1.75 & 0 & 1 & 1 & 0 \\
\hline 224 & 0.028571 & 0.944444 & 1.891892 & 0 & 1 & 1 & 0 & 249 & 0 & 0.5 & 1.125 & 0 & 1 & 1 & 0 \\
\hline 225 & 0.007143 & 0.866667 & 1.891892 & 0 & 1 & 1 & 0 & 250 & 0 & 0.551724 & 1.7 & 0 & 1 & 1 & 0 \\
\hline
\end{tabular}

\begin{tabular}{|c|c|c|c|c|c|c|c|c|c|c|c|c|c|c|c|}
\hline \multirow{2}{*}{ no } & \multicolumn{3}{|c|}{ Input } & \multicolumn{4}{|c|}{ Target } & \multirow{2}{*}{ no } & \multicolumn{3}{|c|}{ Input } & \multicolumn{4}{|c|}{ Target } \\
\hline & $x 1$ & $x 2$ & $x 3$ & $\mathrm{y} 1$ & $y 2$ & $y 3$ & $y 4$ & & $x 1$ & $x 2$ & $x 3$ & $\mathrm{y} 1$ & $y_{2}$ & $y 3$ & $\mathrm{y}^{4}$ \\
\hline 251 & 0 & 0.5 & 1.125 & 0 & 1 & 1 & 0 & 276 & 0.25 & 0.4 & 0.666667 & 0 & 1 & 1 & 1 \\
\hline 252 & 0.5 & 1.6 & 0.111111 & 0 & 1 & 1 & 0 & 277 & 0 & 1.2 & 0.2 & 0 & 1 & 1 & 1 \\
\hline 253 & 0.142857 & 0.176471 & 0.7 & 0 & 1 & 1 & 0 & 278 & 0 & 2 & 0.333333 & 0 & 1 & 1 & 1 \\
\hline 254 & 0 & 1 & \begin{tabular}{|l|}
0.571429 \\
\end{tabular} & 0 & 1 & 1 & 0 & 279 & 0 & 1.2 & 0.2 & 0 & 1 & 1 & 1 \\
\hline 255 & 0.142857 & 1.5 & 3.888889 & 0 & 1 & 1 & 0 & 280 & 0 & 2 & 0.333333 & 0 & 1 & 1 & 1 \\
\hline 256 & \begin{tabular}{|l|}
0.521739 \\
\end{tabular} & 0.095238 & 3.285714 & 0 & 1 & 1 & 0 & 281 & 0 & 0.428571 & 1 & 0 & 1 & 1 & 1 \\
\hline 257 & 0 & 1.2 & 0.25 & 0 & 1 & 1 & 1 & 282 & \begin{tabular}{|l|l|}
0.041667 \\
\end{tabular} & 3.4 & 0.387097 & 0 & 1 & 1 & 1 \\
\hline 258 & 0.2 & 0.285714 & 0.714286 & 0 & 1 & 1 & 1 & 283 & 0.066667 & 7.111111 & 0.105263 & 0 & 1 & 1 & 1 \\
\hline 259 & 0 & 1.3125 & 0.944444 & 0 & 1 & 1 & 1 & 284 & 0 & \begin{tabular}{|l}
6.166667 \\
\end{tabular} & 0.085366 & 0 & 1 & 1 & 1 \\
\hline 260 & 0 & 1.5 & \begin{tabular}{|l|l|}
0.142857 \\
\end{tabular} & 0 & 1 & 1 & 1 & 285 & 0 & 2.666667 & 0.6 & 0 & 1 & 1 & 1 \\
\hline 261 & 0 & 1.692308 & 0.580645 & 0 & 1 & 1 & 1 & 286 & 0.071429 & 4.333333 & 0.142857 & 0 & 1 & 1 & 1 \\
\hline 262 & 0.055556 & 4 & 0.9 & 0 & 1 & 1 & 1 & 287 & 0 & 2.777778 & 0.25 & 0 & 1 & 1 & 1 \\
\hline 263 & 1 & 8.8 & 0.032258 & 0 & 1 & 1 & 1 & 288 & 0 & 7.4 & 0.652174 & 0 & 1 & 1 & 1 \\
\hline 264 & 0.5 & 14.4 & 0.025 & 0 & 1 & 1 & 1 & 289 & 0.5 & 1 & 0.083333 & 0 & 1 & 1 & 1 \\
\hline 265 & 1 & 9 & 0.037037 & 0 & 1 & 1 & 1 & 290 & 0 & 1 & 1 & 0 & 1 & 1 & 1 \\
\hline 266 & 0.083333 & 14.6 & 0.153846 & 0 & 1 & 1 & 1 & 291 & 0 & 1.2 & 0.5 & 0 & 1 & 1 & 1 \\
\hline 267 & 0.25 & 0.285714 & 0.5 & 0 & 1 & 1 & 1 & 292 & 0 & 40.22414 & 0.32 & 0 & 1 & 1 & 1 \\
\hline 268 & 0.166667 & 0.093023 & 1 & 0 & 1 & 1 & 1 & 293 & 0 & 1.2 & 0.5 & 0 & 1 & 1 & 1 \\
\hline 269 & 0.25 & 0.063291 & 1 & 0 & 1 & 1 & 1 & 294 & 0 & 2.777778 & 0.25 & 0 & 1 & 1 & 1 \\
\hline 270 & 0.166667 & 0.4 & 0.428571 & 0 & 1 & 1 & 1 & 295 & 0 & 7.4 & 0.652174 & 0 & 1 & 1 & 1 \\
\hline 271 & 0 & 2.2 & 0.24 & 0 & 1 & 1 & 1 & 296 & 0.5 & 0.666667 & 0.333333 & 0 & 1 & 1 & 1 \\
\hline 272 & 0 & 1.555556 & 0.272727 & 0 & 1 & 1 & 1 & 297 & 0 & 2.208333 & 0.226415 & 0 & 1 & 1 & 1 \\
\hline 273 & 0 & 2.222222 & 0.333333 & 0 & 1 & 1 & 1 & 298 & 0.071429 & 2.777778 & 0.25 & 0 & 1 & 1 & 1 \\
\hline 274 & 0.25 & \begin{tabular}{|l|l|}
0.142857 \\
\end{tabular} & \begin{tabular}{|l|}
0.857143 \\
\end{tabular} & 0 & 1 & 1 & 1 & 299 & 0.5 & 1 & 0.083333 & 0 & 1 & 1 & 1 \\
\hline 275 & 0.5 & \begin{tabular}{|l|}
0.230769 \\
\end{tabular} & 0.8 & 0 & 1 & 1 & 1 & 300 & 0 & 1.111111 & 1.130435 & 1 & 0 & 0 & 0 \\
\hline
\end{tabular}


Hartono, Y. Muharni, C. Adipura, W. Martiningsih, M. Otong, and M. Irvan

\begin{tabular}{|c|c|c|c|c|c|c|c|}
\hline \multirow{2}{*}{ no } & \multicolumn{3}{|c|}{ Input } & \multicolumn{4}{|c|}{ Target } \\
\hline & $x 1$ & $x 2$ & $x 3$ & y1 & $\mathrm{y}^{2}$ & $y_{3}$ & $\mathrm{y} 4$ \\
\hline 301 & 0 & 1.4 & 1.333333 & 1 & 0 & 0 & 0 \\
\hline 302 & 0 & 3.944444 & 2.235294 & 1 & 0 & 0 & 0 \\
\hline 303 & 0.041667 & 2.333333 & 1.090909 & 1 & 0 & 0 & 0 \\
\hline 304 & 0.022727 & 1.130435 & 7.333333 & 1 & 0 & 0 & 1 \\
\hline 305 & 0.019608 & 2.903226 & 15 & 1 & 0 & 0 & 1 \\
\hline 306 & 0.022727 & 3.394737 & 10.42105 & 1 & 0 & 0 & 1 \\
\hline 307 & 0.024194 & 0.784314 & 5.636364 & 1 & 0 & 0 & 1 \\
\hline 308 & 0.013812 & 2.837838 & 13.92308 & 1 & 0 & 0 & 1 \\
\hline 309 & 0.040323 & 2.179487 & 8.266667 & 1 & 0 & 0 & 1 \\
\hline 310 & 0.015625 & 4 & 11.63636 & 1 & 0 & 0 & 1 \\
\hline 311 & 0.030303 & 2.289474 & 16.5 & 1 & 0 & 0 & 1 \\
\hline 312 & 0.045802 & 3.3 & 13.1 & 1 & 0 & 0 & 1 \\
\hline 313 & 0.035088 & 1.666667 & 19 & 1 & 0 & 0 & 1 \\
\hline 314 & 0.04652 & 1.569767 & 11.375 & 1 & 0 & 0 & 1 \\
\hline 315 & 0.030303 & 2.289474 & 16.5 & 1 & 0 & 0 & 1 \\
\hline 316 & 0.023973 & 2.151515 & 13.27273 & 1 & 0 & 0 & 1 \\
\hline 317 & 0.0625 & 1.285714 & 4 & 1 & 0 & 0 & 1 \\
\hline 318 & 1 & 0.333333 & 0.125 & 1 & 0 & 0 & 1 \\
\hline 319 & 0.5 & 1.571429 & 0.125 & 1 & 0 & 0 & 1 \\
\hline 320 & 0.059211 & 1.519231 & 19 & 1 & 0 & 0 & 1 \\
\hline 321 & 0.077778 & 1.571429 & 20.57143 & 1 & 0 & 0 & 1 \\
\hline 322 & 0.003597 & 1.702128 & 13.9 & 1 & 0 & 0 & 1 \\
\hline 323 & 0.291026 & 1.128205 & 11.14286 & 1 & 0 & 0 & 1 \\
\hline 324 & 0.030303 & 5.571429 & 8.608696 & 1 & 0 & 0 & 1 \\
\hline 325 & 0.071429 & 4 & 3.5 & 1 & 0 & 0 & 1 \\
\hline 326 & 0.1 & 4.2 & 2.5 & 1 & 0 & 0 & 1 \\
\hline 327 & 0.166667 & 3.8 & 2 & 1 & 0 & 0 & 1 \\
\hline 328 & 1.5 & 4.2 & 0.333333 & 1 & 0 & 0 & 1 \\
\hline 329 & 0.5 & 0.666667 & 0.333333 & 1 & 0 & 0 & 1 \\
\hline 330 & 0.071429 & 4 & 3.5 & 1 & 0 & 0 & 1 \\
\hline 331 & 0.1 & 4.2 & 2.5 & 1 & 0 & 0 & 1 \\
\hline 332 & 0.03989 & 4.391753 & 11.66667 & 1 & 0 & 0 & 1 \\
\hline 333 & 0.007417 & 0.022348 & 4.993827 & 1 & 0 & 0 & 1 \\
\hline 334 & 0.071429 & 2.081081 & 3.5 & 1 & 0 & 0 & 1 \\
\hline 335 & 0.071429 & 4 & 3.5 & 1 & 0 & 0 & 1 \\
\hline 336 & 0.166667 & 3.8 & 2 & 1 & 0 & 0 & 1 \\
\hline 337 & 0.020548 & 2.611111 & 10.42857 & 1 & 0 & 0 & 1 \\
\hline 338 & 0.077778 & 1.571429 & 20.57143 & 1 & 0 & 0 & 1 \\
\hline 339 & 2.05 & 0.010959 & 6.5 & 1 & 0 & 0 & 1 \\
\hline 340 & 0.125 & 0.714286 & 0.444444 & 1 & 0 & 0 & 1 \\
\hline 341 & 0.060734 & 2.485714 & 16.85714 & 1 & 0 & 0 & 1 \\
\hline
\end{tabular}

\section{SOURCES OF FUNDING}

None.

\section{CONFLICT OF INTEREST}

None.

\section{ACKNOWLEDGMENT}

I give thanks to God Almighty, because of His blessings and grace, I was able to complete this thesis. This research writing is carried out in order to fulfill one of the requirements to obtain an reasearch in the Department of Electrical Engineering at the Sultan Ageng Tirtayasa University. I realize that, without the help and guidance of various parties, from the lecture period to when writing this research, discussing it was difficult for me to complete this research.

\section{REFERENCES}

[1] Gedam, A. V, Swami, P. P. S., \& Thosar, A. (2015). A-Comparative-Analysis-of-DGA-Methods-For-The-IncipientFault-Diagnosis-in-Power-Transformer-Using-ANN-Approach.docx. 6(5), 387-391.

[2] Sharma, N. K., Tiwari, P. K., \& Sood, Y. R. (2011). Review of Artificial Intelligence Techniques Application to Dissolved Gas Analysis on Power Transformer. International Journal of Computer and Electrical Engineering, 3(4), 577-582. https://doi.org/10.7763/ijcee.2011.v3.383 
Analysis of Power Transformator Conditions Using DGA Method Using Artificial Neural Network in Krakatau Electrical Power Company

[3] Nourmohammadzadeh, A., \& Hartmann, S. (2015). Fault Classification of a Centrifugal Pump in Normal and Noisy Environment with Artificial Neural Network and Support Vector. Fourth International Conference on Theory and Practice of Natural Computing, 1, 58-70. https://doi.org/10.1007/978-3-319-26841-5

[4] Hartono, \& Kuo, M.-T. (2018). Design of Simulation forTransient Stability Analysisin Smart Grid by Using Critical Clearing Time Index. International Journal of Engineering and Technology, 10(3), 269-273. https://doi.org/10.7763/ijet.2018.v10.1072

[5] Moosavian, A., Ahmadi, H., Tabatabaeefar, A., \& Khazaee, M. (2013). Comparison of two classifiers; K-nearest neighbor and artificial neural network, for fault diagnosis on a main engine journal-bearing. Shock and Vibration, 20(2), 263-272. https://doi.org/10.3233/SAV-2012-00742

[6] Yoru, Y., Karakoc, T. H., \& Hepbasli, A. (2009). Application of Artificial Neural Network (ANN) method to exergy analysis of thermodynamic systems. 8th International Conference on Machine Learning and Applications, ICMLA 2009, 715-718. https://doi.org/10.1109/ICMLA.2009.70

[7] Nagpal, T., \& Brar, Y. S. (2014). Artificial neural network approaches for fault classification: comparison and performance. Neural Computing and Applications, 25(7-8), 1863-1870. https://doi.org/10.1007/s00521014-1677-y

[8] Ghoneim, S. S. M., \& Taha, I. B. (2015). Artificial Neural Networks for Power Transformers Fault Diagnosis Based on IEC Code Using Dissolved Gas Analysis. International Journal of Control, Automation and Systems, $4(2), 18-21$.

[9] Saranya, S., Mageswari, U., Roy, N., \& Sudha, R. (2013). Comparative Study of Various Dissolved Gas Analysis Methods to Diagnose Transformer Faults. International Journal of Engineering Research and Applications (IJERA), 3(3), 592-595. www.ijera.com

[10] Pereira, F. H., Bezerra, F. E., Junior, S., Santos, J., Chabu, I., De Souza, G. F. M., Micerino, F., \& Nabeta, S. I. (2018). Nonlinear autoregressive neural network models for prediction of transformer oil-dissolved gas concentrations. Energies, 11(7). https://doi.org/10.3390/en11071691

[11] Roland, U., \& Eseosa, O. (2015). Artificial Neural Network Approach to Distribution Transformers Maintenance. International Journal of Scientific Research Engineering Technology (IJSRET), 1(4), 62-70.

[12] Yu, S., Zhao, D., Chen, W., \& Hou, H. (2016). Oil-immersed Power Transformer Internal Fault Diagnosis Research Based on Probabilistic Neural Network. Procedia Computer Science, 83(Wtisg), 1327-1331. https://doi.org/10.1016/j.procs.2016.04.276

[13] Hartono, H., Marifa Ahmad, A., \& Sadikin, M. (2018). Comparison methods of short-term electrical load forecasting. MATEC Web of Conferences, 218, 1-8. https://doi.org/10.1051/matecconf/201821801002

[14] Muthi, A., Sumarto, S., \& Saputra, W. S. (2018). Power Transformer Interruption Analysis Based on Dissolved Gas Analysis (DGA) using Artificial Neural Network. IOP Conference Series: Materials Science and Engineering, 384(1), 0-5. https://doi.org/10.1088/1757-899X/384/1/012073

[15] Rigatos, G., \& Siano, P. (2016). Power transformers' condition monitoring using neural modeling and the local statistical approach to fault diagnosis. International Journal of Electrical Power and Energy Systems, 80, 150159. https://doi.org/10.1016/j.ijepes.2016.01.019

[16] H. D. Mehta, R. M. P. (2014). A Review on Transformer Design Optimization and Performance Analysis Using Artificial Intelligence Techniques. International Journal of Science and Research (IJSR), 3(9), https://www.ijsr.net/archive/v3i9/U0VQMTQxOTg=.pdf

[17] Adeolu, O., \& Adejumobi, I. A. (2014). Breakdown Voltage Characteristics of Castor Oil as Alternative to Transformer Insulation Oil. 2(4), 31-37.

[18] Al-Janabi, S., Rawat, S., Patel, A., \& Al-Shourbaji, I. (2015). Design and evaluation of a hybrid system for detection and prediction of faults in electrical transformers. International Journal of Electrical Power and Energy Systems, 67, 324-335. https://doi.org/10.1016/j.ijepes.2014.12.005

[19] G. K. M. A. P. C. Pravin S. Khade, "Artificial Neural Netwok Approach to Dissolved Gas Analysis for Interpretation of fault in power transformer," International journal Of Sctentific \& Engineering Reasearch, no. 373-377. 